\title{
MAIA, a three-channel imager for asteroseismology: instrument design ${ }^{\star}$
}

\author{
Gert Raskin $^{1}$, Steven Bloemen ${ }^{1,4}$, Johan Morren ${ }^{1}$, Jesus Perez Padilla ${ }^{1,2}$, Saskia Prins ${ }^{1,2}$, Wim Pessemier ${ }^{1}$, \\ Jeroen Vandersteen ${ }^{3}$, Florian Merges ${ }^{1,2}$, Roy Østensen ${ }^{1}$, Hans Van Winckel ${ }^{1}$, and Conny Aerts ${ }^{1,4}$ \\ ${ }^{1}$ Institute of Astronomy, Department of Physics and Astronomy, KU Leuven, Celestijnenlaan 200D, 3001 Leuven, Belgium \\ e-mail: gert.raskin@ster. kuleuven.be \\ 2 Mercator Telescope, Roque de los Muchachos Observatory, 38700 Santa Cruz, La Palma, Spain \\ ${ }^{3}$ RHEA, European Space Agency - ESTEC, Keplerlaan 1, 2201 AZ Noordwijk, The Netherlands \\ ${ }^{4}$ Department of Astrophysics, IMAPP, Radboud University Nijmegen, PO Box 9010, 6500 GL Nijmegen, The Netherlands
}

Received 10 August 2013 / Accepted 8 October 2013

\begin{abstract}
MAIA, an acronym for Mercator Advanced Imager for Asteroseismology, is a three-channel instrument that targets fast-cadence threecolour photometry, installed at the 1.2-m Mercator telescope at the Roque de los Muchachos at La Palma (Canary Islands, Spain). This instrument observes a $9.4 \times 14.1 \mathrm{arcmin}^{2}$ field-of-view simultaneously in three wavelength bands on three large frame-transfer CCDs. These detectors were developed for ESA's cancelled Eddington space mission and were offered on permanent loan to the Institute of Astronomy (KU Leuven, Belgium). MAIA uses its own ugr photometric system that is a crude approximation of the SDSS system. The instrument is designed to perform multi-colour observations for asteroseismology, with specific emphasis on subdwarf and white dwarf single and binary stars. We describe the design of the instrument, discuss key components, and report on its performance and first results.
\end{abstract}

Key words. asteroseismology - instrumentation: photometers - instrumentation: detectors - techniques: photometric stars: variables: general

\section{Introduction}

The MAIA project found its origin in the Eddington space mission that was cancelled by the European Space Agency (ESA) in 2003. The main objectives of Eddington were asteroseismology and exoplanet transit detections (Favata et al. 2000). A number of charge-coupled device (CCD) detectors had been developed and procured specifically for this mission, based on the wellknown e2v CCD42-xx series. These are large frame-transfer devices, designated as CCD42-C0.

After the cancellation of the Eddington mission, some of these detectors were offered to the astronomical community provided that they would be used for the original science goals of the cancelled Eddington mission. One of us (CA) defined a proposal to use the CCDs to perform asteroseismic studies of evolved stars, in particular of subdwarf B (sdB) stars, by constructing a camera to be installed at the 1.2-m Mercator telescope. As such, the Institute of Astronomy of the University of Leuven (KULeuven, Belgium) received the Eddington detectors through a permanent loan agreement between ESA and KU Leuven. The present paper presents the scientific specifications, design, installation and the first performance tests of the Mercator Advanced Imager for Asteroseismology (MAIA).

The Mercator telescope (Raskin et al. 2004) is a modern, semi-robotic 1.2-m telescope, installed at the Roque de los Muchachos Observatory on La Palma (Canary Islands, Spain),

* Based on observations made with the Mercator Telescope, operated on the island of La Palma by the Flemish Community, at the Spanish Observatorio del Roque de los Muchachos of the Instituto de Astrofísica de Canarias. funded by the Flemish community of Belgium, and operated by the KU Leuven Institute of Astronomy. This telescope provides Belgian astronomers with permanent access to an intermediatesize telescope, opening up the niche of high-precision long-term studies of time-variable astronomical phenomena. This has allowed the Leuven team and its collaborators to continue its leading role in the field of variable single and multiple star research, where long time-series are essential. The operational model of Mercator is focused on and committed to long-term programmes which are particularly important for the study of stellar pulsations, where the time span of the observations defines the precision with which independent pulsation frequencies can be detected and identified. Another prime science case for the Mercator telescope is the study of binary stars, with specific emphasis on long-period evolved binaries where orbits of one to several years are commonly found.

Currently, the Mercator telescope is also equipped with a stable and efficient high-resolution échelle spectrograph (HERMES, Raskin et al. 2011). The MEROPE imager (Davignon et al. 2004) was already equipped with one of the Eddington detectors from 2009 until 2012 for performance tests and characterisation purposes in preparation for MAIA (MEROPE II, Østensen 2010), and has since been decommissioned in order to install MAIA.

The preliminary design of MAIA was first presented in Vandersteen et al. (2010). In the meantime, we have built the instrument, installed it on the telescope (2012) and almost completed the commissioning (to be finished by the end of 2013). In this paper, we discuss the scientific motivation behind the project and its consequences for the instrument requirements, 
as well as a detailed description of its design. We also report on the measured performance of MAIA and present some first onsky results. In a subsequent paper (Bloemen et al., in prep.), we plan to present the detailed commissioning of the instrument, the data reduction software necessary for the optimal scientific use of the instrument, and illustrate its capacity for asteroseismology of pulsating subdwarf B stars.

\section{Science case}

Putting efforts into ground-based asteroseismology remains very relevant for particular types of stars, even if the space missions MOST (Walker et al. 2003), CoRoT (Auvergne et al. 2009) and Kepler (Gilliland et al. 2010) have resulted in a revolution in our knowledge of stellar interiors. Indeed, while the $\mu$ mag precision white-light space photometry led to various recent breakthroughs to improve stellar physics, e.g. the ability to deduce inhomogeneously mixed zones around the convective core of massive stars (e.g. Degroote et al. 2010), the connection between beating of oscillation modes and outbursts in Be stars (e.g. Huat et al. 2009), the discovery of gravito-inertial modes in fast rotators (e.g. Neiner et al. 2012; Pápics et al. 2012), the power to discriminate between hydrogen-shell and core-helium burning red giants from their dipole mixed modes (e.g. Bedding et al. 2011), the derivation of core to envelope rotation of subgiants (Deheuvels et al. 2012) and of red giants (Beck et al. 2012), to list just a few, some types of pulsators could not be studied, or insufficiently so, by these missions. This is particularly the case for ultra-fast pulsators, such as the roAp stars, pressure-mode pulsating subdwarfs and gravity-mode pulsating white dwarfs, with pulsation periods of the order of one to a few minutes and of which too few class members occurred in the field-of-view (FoV) of the satellites to do ensemble asteroseismology of those classes. MAIA was designed to be able to target asteroseismology of such fast and relatively faint pulsators.

A particular point of attention, bridging two important scientific aims of the Mercator telescope which cannot be done with a space missions alone, was to build an instrument to complement the HERMES spectrograph for the study of binary pulsators that underwent a phase of common envelope or stable Roche-lobe overflow during the red-giant branch. Presently, the physics of the common envelope phase is still described by an ad-hoc parameter connected with the envelope ejection efficiency. In this model, it is assumed that the orbital energy released during the spiral-in is used to eject the common envelope of the two stars and results in a short-period binary in the core-helium burning phase. In the case of stable Roche-lobe overflow, one expects to find long-period horizontal branch binaries but hardly any have been found so far due to the long-term monitoring requirement (e.g. Vos et al. 2012). Luckily, pulsators on the horizontal branch that have passed these poorly known evolutionary stages exist and these allow seismic tuning of their envelope structure and mass, and subsequent backtracking of their previous evolution (e.g. Hu et al. 2008). Despite impressive results on horizontal branch star asteroseismology from Kepler (e.g. Van Grootel et al. 2010; Charpinet et al. 2011; Reed et al. 2011; Østensen 2013), only a few Kepler targets are suitable to tackle the binary evolution case (Østensen et al. 2010; Pablo et al. 2012).

In general, asteroseismology allows one to constrain the internal structure of stars by means of forward modelling, starting from a frequency analysis and secure identification of the spherical degree $\ell$ of several detected pulsation modes. Such forward modelling delivers the stellar fundamental parameters with typically an order of magnitude better precision than can be achieved from the comparison of classical data, such as spectrum analysis and/or interferometry, with stellar models. Moreover, asteroseismology allows, in principle, to improve the input physics of the current stellar models by exploiting the seismic information in full details, as has been achieved for the Sun (e.g. Christensen-Dalsgaard 2002).

A prerequisite for a successful seismic application, is an unambiguous identification of the detected pulsation modes. Three well established methods for identifying the modes of a pulsating star are available:

1. from white light or single band photometry by means of recognition of frequency or period spacings for modes of consecutive radial orders, or from rotationally split multiplets;

2. from multi-band photometry, employing simultaneous observations in at least three different wavelength bands, through interpretation of the measured amplitude ratios and/or phase differences;

3. from time-resolved high signal-to-noise spectroscopy allowing the line-profile variations of unblended spectral lines to be interpreted

(e.g. Aerts et al. 2010, Chap. 6). MAIA is designed to use method 2 in the case of relatively faint and fast pulsators with amplitudes near $100 \mu$ mag or higher, whose binarity can be modelled from long-term HERMES spectroscopy. However, since this particular science case is more demanding than seismic applications to most other classes of pulsators with similar amplitudes, it can also treat various other cases, and in particular stars where space asteroseismology was not possible or very limited so far, e.g., hot massive supergiant asteroseismology.

\section{Instrument requirements}

The following high-level instrument requirements could be derived from the science case that was presented in the previous section:

1. Simultaneous measurements in at least three optical wavelength bands. To increase the contrast of the amplitude ratios, the separation between the dichroic cut points should be as wide as possible.

2. High sample rate. For accurate sampling of the shortest pulsation periods, cycle times as short as a few seconds are required. Obtaining acceptable exposure times in such short periods means that the dead time between exposures should be negligible.

3. Uninterrupted long-term availability. The exploitation scheme of the Mercator telescope foresees to have all instruments permanently installed. Switching between instruments only takes a few minutes. Consequently, MAIA is almost permanently available for observations. This is essential for obtaining accurate oscillation frequencies as they require continuous time series of observations with a long time base.

4. Wide field-of-view. Precise measurements of the brightness variation of pulsating stars require the simultaneous observation of preferably several reference stars. Especially in the $u$ band, it is difficult to find bright enough comparison stars. To increase the probability of finding sufficiently bright reference sources, MAIA should cover a large FoV. The MEROPE II camera on the Mercator telescope had a FoV of $9 \times 6.6 \mathrm{arcmin}^{2}$. Our experience with this instrument 
showed that MAIA should cover a FoV that is at least twice as large.

5. Spatial resolution. MAIA image quality should only be a negligible contributor to the point spread function (PSF), defined by the size of the seeing disk in case of excellent atmospheric conditions at the Roque De Los Muchachos observatory on La Palma ( $\sim 0.6$ arcsec). The sampling of an 0.6-arcsec PSF should satisfy the Nyquist criterion.

6. High throughput. Obviously, high sample rates go hand in hand with short exposure times. However, many possible MAIA targets are faint or span a broad brightness range. This is especially relevant when considering the different wavelength ranges that are sampled simultaneously. For a typical reference star, the $u$-band flux can be very small and many magnitudes fainter than the $r$ or $g$-band flux. Given the limited collecting area of the 1.2-m Mercator telescope, it is of high importance to reduce to an absolute minimum the number of photons that get lost on their way through the instrument.

7. Portability. At the time of writing, no concrete plans exist to take MAIA to other telescopes than Mercator. Nevertheless, in order to extend MAIA's scope towards fainter targets, we also consider deploying the instrument at larger aperture telescopes in the future. Therefore, MAIA should be designed with portability in mind and the optics should be adaptable to a telescope that is at least twice as large as the 1.2-m Mercator telescope.

8. Detectors. The budget for building MAIA leaves no room to procure dedicated detectors and hence relies completely on the use of the Eddington CCD42-C0 devices provided by ESA. Therefore, the instrument design has to be adapted to the properties of these detectors. Fortunately, the pixel size and the pixel count of the Eddington CCDs are well suited for the type of observations targeted by MAIA (see Sect. 4.1.1).

\section{Instrument design}

The design of MAIA is loosely inspired by ULTRACAM (Dhillon et al. 2007) but in contrast to this instrument, MAIA does not target sub-second sample rates. Due to the much larger detectors, however, MAIA offers a much larger FoV. In the following sections, we present the most important design aspects of MAIA.

\subsection{Detector system}

\subsubsection{The CCD42-C0 frame-transfer detectors}

The starting point of the MAIA design are the CCD42-C0 detectors that were developed by e2v (UK) for the Eddington space mission (Lumb \& Favata 2003). These are thinned backilluminated frame-transfer (FT) devices with a basic mid-band anti-reflection coating. Unfortunately, this coating has a rather poor UV response. An enhanced broad-band coating with higher throughput at short wavelengths would have been a much better match for the important MAIA $u$ channel. As a result, the UV quantum efficiency (QE) is much lower than expected (less than $20 \%$ for wavelengths below $370 \mathrm{~nm}$, see Fig. 3). The chips have a format of $2048 \times 614413.5-\mu \mathrm{m}$ pixels, split in halves between a $2 \mathrm{k} \times 3 \mathrm{k}$ imaging area, where charge collection takes place, and an equally large storage area. The effective area of these unique detectors is substantially larger than that of any other commercially available FT device. Figure 1

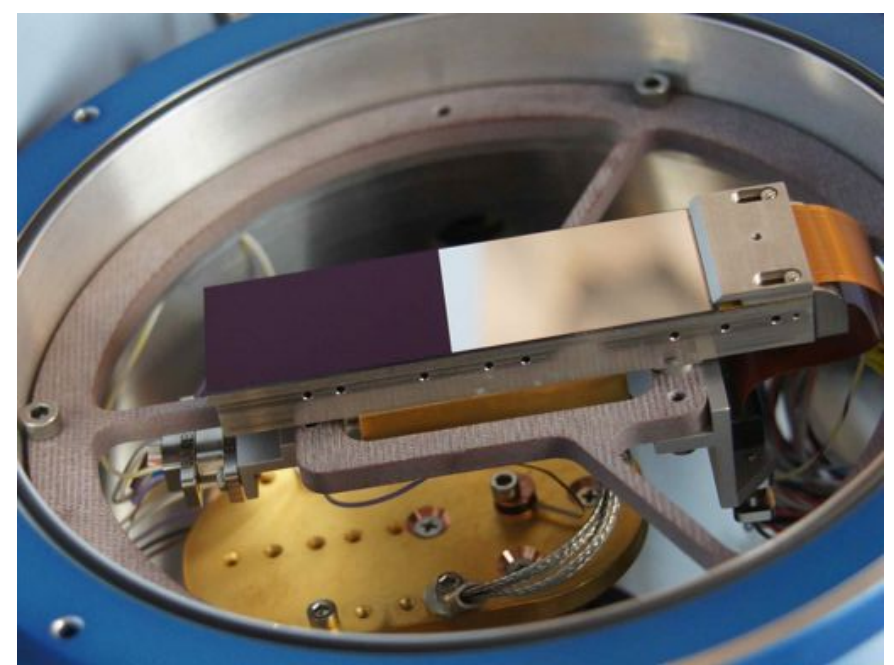

Fig. 1. CCD42-C0 frame-transfer detector, mounted in the $u$ cryostat.

shows a picture of a CCD42-C0 detector, mounted on a fibreglass spider in its cryostat. The dark part at the left-hand side is the photo-sensitive imaging area and the shiny part at the right is the aluminium-covered storage area. The detector is very long $(\sim 110 \mathrm{~mm})$ so it is installed with the imaging area out of centre, in order to reduce the diameter and volume of the cryostat. Consequently, the window in the cryostat is similarly decentred.

The FT design allows a rapid shift of the image data from the imaging to the storage area, so that integration can proceed in the imaging area while the previous image is being digitised from the storage area. The frame transfer shift takes only $295 \mathrm{~ms}$, while the image data readout of a full frame takes between 43 and $30 \mathrm{~s}$, depending on the controller digitisation speed settings and the read-out noise requirements (see Table 1 ). The time required to transfer a full-frame image from the imaging to the storage area is more than 100 times smaller than the full-frame read-out time. The dead time during time-series observations is only limited to this $295 \mathrm{~ms}$ frame transfer. The usage of the FT mode implies that the integration time should be at least as long as the duration of the image read-out from the store area. When shorter integration times are required, data can also be acquired using a classic read-out mode with a destructive clear prior to the integration, but in that case the full read-out overhead applies.

All three MAIA cameras are equipped with a mechanical, individually controlled, iris shutter that allows the detectors to be used in classic (non-FT) mode. In FT mode the shutters are continuously open.

\subsubsection{Detector cooling}

Although MAIA's main science case only needs short integration times, instrument versatility also required the possibility of obtaining long exposures. Even when observing the faintest targets, detector dark current should remain a negligible noise source. This means that it should be significantly smaller than the flux from the new-moon sky, which can be as small as $0.1 \mathrm{e}^{-}$pixel $^{-1} \mathrm{~s}^{-1}$ in the $u$ band for MAIA on the Mercator telescope. In this case, the dark current should not exceed $0.05 \mathrm{e}^{-}$pixel $^{-1} \mathrm{~s}^{-1}$. Laboratory measurements show that the CCD42-C0 needs a temperature below $190 \mathrm{~K}$ to reach this dark current level. Alternatively, operating the detector in inverted state would reduce the dark current at a much higher temperature. However, a negative consequence of this inversion is a 
substantially reduced full-well capacity. This seriously limits the dynamic range of the cameras so we discarded this solution.

We explored various technologies to cool the MAIA detectors to $190 \mathrm{~K}$. Thermo-electric cooling of very large detectors like the CCD42-C0 by multi-stage Peltier elements, turned out to be extremely difficult. Moreover, in the absence of cryogenic temperatures, cryo-pumping could not be used to maintain a long-term vacuum in the cryostat. Liquid nitrogen $\left(\mathrm{LN}_{2}\right)$ and Joule-Thompsom (JT) cooling with e.g. a CryoTiger system are long-time proven solutions. However, the use of $\mathrm{LN}_{2}$ was discarded because of the burden of filling three cryostats at the start and end of each night. JT coolers for three cryostats implied two or three compressors and six gas lines with heavy stainless steel braiding, a very bulky system to accommodate at the Nasmyth focal station of Mercator. We therefore looked for an alternative and settled on the use of compact free-piston Stirling coolers to cool the MAIA detectors. After some market research, we selected the MT CryoTel cryocooler (Sunpower, USA) that can evacuate $5 \mathrm{~W}$ of heat at $77 \mathrm{~K}$ with an electrical input power of $80 \mathrm{~W}$ (Unger \& Keiter 2004). These are very convenient and compact devices with a weight of only $2.1 \mathrm{~kg}$. The vibrations generated by the linear compressor are the only important disadvantage. In Sect. 4.3.2 we discuss how these vibrations are reduced to an acceptable level.

The thermal link between the detector and the cold head of the cooler is dimensioned to obtain a detector equilibrium temperature around $155 \mathrm{~K}$. A resistive heater in closed loop heats the detector and stabilises its temperature at $165 \mathrm{~K}$ with an accuracy of a few $0.01 \mathrm{~K}$. We use a programmable logic controller (PLC) and industrial hardware to implement this feedback loop, as well as for all other instrument control tasks (Pessemier et al. 2012). A human-machine interface (HMI) with touch screen is mounted on the instrument. It provides a visual indication of the status (e.g. detector temperature, cooling power, etc.) and allows manual technical control of MAIA (e.g. changing temperature setpoint, starting a controlled warm-up procedure, etc.).

\subsubsection{Data acquisition}

The three MAIA detectors are read out and controlled by a standard generation-III SDSU detector controller (Astronomical Research Cameras, USA) (Leach \& Low 2000). Volume and weight restrictions required the use of the small 6-slot housing and small power supply. Therefore, the controller could only be equipped with two dual channel video boards, and thus only four read ports can be used. Hence, although the detectors have a dual-port split serial register, only single-port read-out is currently implemented. Windowing and binning are used to reduce the read time if short exposure times are required.

We have implemented standard single windowing of the detectors as well as a multi-window mode. In the multi-window mode, up to ten windows can be defined anywhere on the detector. All windows must have the same dimensions. The choice of window height and vertical position is free but the windows should not overlap. We found that serial skips between prescan, science windows and overscan, cause a disturbing bias level gradient of several digitisation units at the horizontal start of each window. Therefore, the horizontal extent of the windows was fixed to the full width of the detector, including prescan and overscan (2150 pixels).

In Table 1, characteristics of the two main detector read-out modes are given. In case the detectors are operated in frametransfer mode, the minimum exposure time is defined by the read-out time. When combining the frame-transfer mode with
Table 1. MAIA detector read-out modes. Read noise is slightly lower in $u$ and $r$ than it is in $g$.

\begin{tabular}{lcc}
\hline \hline Read-out mode & Slow & Fast \\
\hline Read-out frequency & $152 \mathrm{kPixel} \mathrm{s}^{-1}$ & $219 \mathrm{kPixel} \mathrm{s}^{-1}$ \\
Read-out noise & $3.5-4 \mathrm{e}^{-}$ & $4.5-5 \mathrm{e}^{-}$ \\
Single row read-out time & $14.1 \mathrm{~ms}$ & $9.8 \mathrm{~ms}$ \\
Full frame read-out time & $43.5 \mathrm{~s}$ & $30.3 \mathrm{~s}$ \\
Full frame transfer time & $295 \mathrm{~ms}$ & $295 \mathrm{~ms}$ \\
Conversion gain & $0.8 \mathrm{ADU} / \mathrm{e}^{-}$ & $0.4 \mathrm{ADU} / \mathrm{e}^{-}$ \\
\hline
\end{tabular}

windowing of the detector, the single-row read-out time can be used to determine the read-out and minimum exposure times by multiplying with the number of window rows. As an example, two windows of 60 rows each (one placed at the top and one at the bottom part of the detector, both spanning the entire detector width), require a minimum cycle time of two seconds in the most common Slow read-out mode.

A combination of the lower instrumental efficiency in the $u$ band, the often smaller fluxes due to the intrinsic energy distribution of the sources and the high extinction of the earth atmosphere at blue wavelengths, means that saturation can occur in the $g$ or $r$ channel before a sufficiently high signal-to-noise ratio is obtained in $u$. Therefore, we modified the code for the timing-board digital signal processor (DSP) to allow different integration times for the individual channels. After defining a nominal integration time that needs to be longer than the minimal exposure time, each detector can integrate for $1 \times, 2 \times$, or $4 \times$ the nominal integration time. Read-out from the storage area occurs simultaneously for all detectors at every nominal integration time, but image data are only shifted into the storage area at the defined multiples of the integration times.

Currently, all frames obtain a time stamp, based on a computer clock that is synchronised through network time protocol (NTP) with the stratum 2 time server of the observatory. This time stamp may suffer from several variable delays up to $100 \mathrm{~ms}$ due to varying load on the network and the data-acquisition host. Because this is not the most reliable way to ensure accurate timing, we plan to upgrade the Mercator telescope control system with a directly-connected GPS time server in the near future. This time server will also be linked to the MAIA detector controller in order to synchronise the exposures and to deliver reliable and accurate time stamps.

\subsection{Optical design}

\subsubsection{Layout}

A ray trace, illustrating the operation of the MAIA optics, is given in Fig. 2. The light coming from the telescope is first collimated and then split in different wavelength bands by three dichroic beam splitters. Each dichroic splits off one of the three science beams. Three cameras re-image the separated beams on the corresponding detectors. Additional filters can be inserted between the dichroics and cameras for a better definition of the pass bands. Only the reddest light passes through all three dichroics and is lost for science measurements. This near-infrared part of the beam $(\lambda>875 \mathrm{~nm})$ is collected by the MAIA guiding camera to be used for on-axis telescope guiding. As the Mercator telescope does not possess a guiding facility, each instruments needs to be equipped with an integrated guiding camera. 


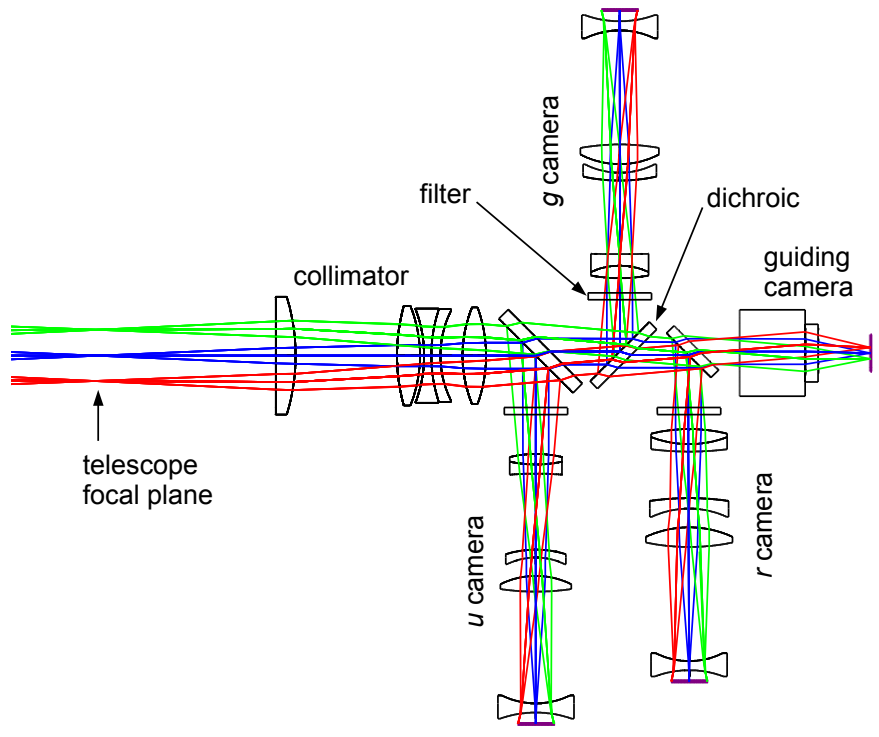

Fig. 2. MAIA optical layout and ray trace. The $r$ channel orientation is perpendicular to the collimator- $u-g$ plane but for clarity, it is $90^{\circ}$ rotated in the drawing.

\subsubsection{Dichroics and filters}

The dichroic beam splitters consist of a flat fused-silica substrate with a dielectric coating that transmits the wavelengths longer than the cut-off point and reflects those that are shorter. For efficiency reasons, the coating is applied to the front surface of the beam splitters, while the rear surface is treated with a $1 \%$ anti-reflection coating. Originally, it was foreseen that the dichroic cut-points would mimic the SDSS photometric system. However, due to a manufacturing problem, the cut-off wavelengths turned out to be slightly longer than foreseen. Moreover, due to the low detector QE in $u$, MAIA has much lower throughput in this band than the SDSS system. The measured 50\% cut points for unpolarised light are given in Table 2. Figure 3 (top) shows the measured transmission curves of the dichroics, together with the transmission of the other optical elements as specified. The dichroics were procured from CVI-Melles Griot (Isle of Man).

Dichroic beam splitters are never perfect components, and a few percent of the flux might end up in the wrong channel. Especially the red light that gets reflected towards the $u$ camera can greatly compromise accuracy. Therefore, additional bandpass filters follow each dichroic. SDSS $u^{\prime}$ and $g^{\prime}$ filters are installed by default in the $u$ and $g$ channels. The MAIA $r$ filter is a short-pass filter that cuts off at $700 \mathrm{~nm}$, substantially below the dichroic upper cut point of $875 \mathrm{~nm}$. This way, we avoid that strong telluric line fluctuations compromise photometric precision. All filters block the wavelengths outside the pass band to less than $0.01 \%$. Figure 3 (bottom) shows the measured transmission of the filters, as well as the total throughput of MAIA, including all optical elements and the detectors.

Filter holders for installing different filters are available. All MAIA filters have a $50.0-50.8 \mathrm{~mm}$ diameter and a maximum thickness of $6 \mathrm{~mm}$. The filter holders need manual insertion in the instrument and therefore, it is not recommended to change filters during an observing night. As the filters are installed in a collimated beam, their absence or different thickness will not affect the focus of the cameras.
Table 2. MAIA dichroic 50\% cut points and effective central wavelength of each filtered pass band.

\begin{tabular}{lcc}
\hline \hline Band & $\lambda_{\text {dichroic cut }}$ & $\lambda_{\text {central }}$ \\
\hline$u$ & $395 \mathrm{~nm}$ & $364 \mathrm{~nm}$ \\
$g$ & $574 \mathrm{~nm}$ & $483 \mathrm{~nm}$ \\
$r$ & $875 \mathrm{~nm}$ & $632 \mathrm{~nm}$ \\
\hline
\end{tabular}

\subsubsection{Collimator and cameras}

To obtain a sufficiently large FoV on the CCD42-C0 detectors, the collimator-camera combination needs to provide some focal reduction. For a FoV of $14.1 \times 9.4 \mathrm{arcmin}^{2}$, a ratio of 1.44 is required between the collimator focal length and the camera focal length. The focal length of the collimator was set at $f=230 \mathrm{~mm}$, a compromise between limited overall dimensions, requiring short focal length, and limited field angles in collimated space, leading to small optical components but requiring long focal length. This gives a focal length of $160 \mathrm{~mm}$ for the cameras. The plate scale at the camera focal plane is then 0.276 arcsec pixel $^{-1}$, providing sufficient sampling under good seeing conditions.

The collimator is a 5 lens system ( 4 singlets and 1 doublet) with an $f / 12$ focal ratio, corresponding to the Mercator telescope optics. The design was driven by having an exit-pupil quite far behind the last collimator lens. This locates the pupil close or inside the cameras, simplifying the camera design and greatly reducing the size of dichroics and camera lenses. The collimator has not been designed to operate as an independent system, but the quality of the collimated beam is more than sufficient to avoid ghost-image issues that would result from a non-parallel beam passing through the thick $45^{\circ}$ dichroics. Instead, optimisation was done on the complete telescope-collimator-camera combination. This allowed us to benefit from additional degrees of freedom without having the image quality of the collimator as a constraint.

All three cameras have a similar design consisting of one doublet and three singlet lenses. The last lens is a field flattener that corrects the combined field curvature from telescope, collimator and camera. The field lens also acts as the vacuum seal of the cryostat. This avoids the need for an additional plane vacuum window and thus reduces Fresnel reflections. To limit instrument dimensions, the three cameras do not have identical pupil locations. For the $u$ channel the pupil sits almost in the centre of the camera, the $g$ pupil lies on the first camera lens, and $r$ has the pupil on the dichroic in front of the camera. The camera focal length of $160 \mathrm{~mm}$ corresponds with a slow $f / 8.3$ focal ratio. The camera apertures have been oversized by about $33 \%$ to decrease the FoV reduction when using MAIA on telescopes larger than the Mercator telescope. Nevertheless, due to the favourable location of the exit pupil of the collimator, the diameters of the camera lenses do not become larger than about $50 \mathrm{~mm}$. All MAIA lenses were manufactured and coated by Optique Fichou (France). The dimensions of the dichroic beam splitters are also adapted to the increased camera aperture. Deploying MAIA at a larger telescope, only requires the replacement of the collimator optics and the mechanical telescope interface ring.

The theoretical image quality of MAIA is excellent over the entire FoV. Figure 4 shows the diameters that encircle $80 \%$ of the energy radiated by a point source, a criterion slightly more strict than the full width at half maximum (FWHM), as a measure for the width of the point spread function. Hardly any degradation of the spatial resolution is expected over most of the FoV, 

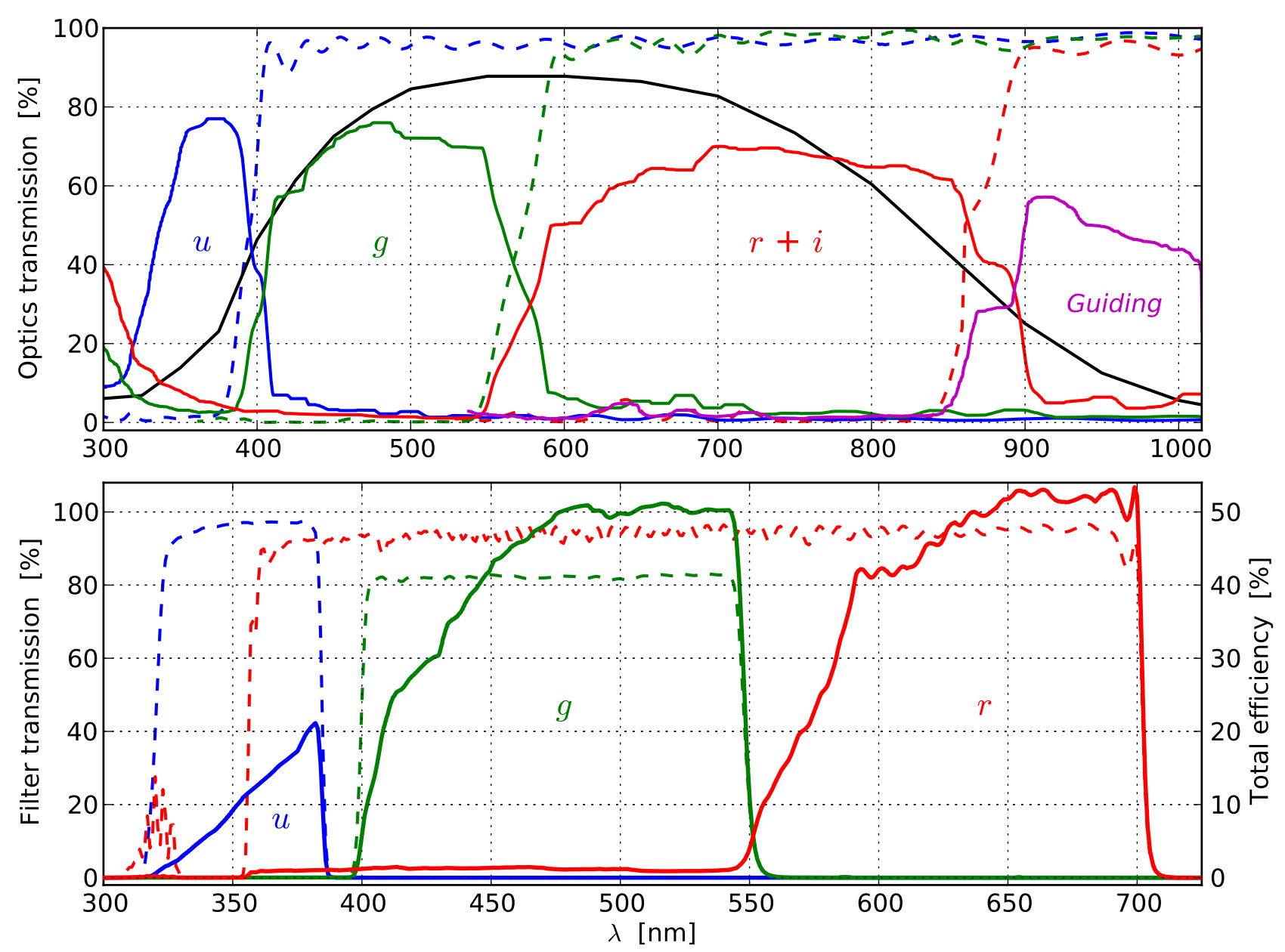

Fig. 3. Top: detector QE (solid black curve), dichroic transmission (dashed colours) and total optics transmission (convolution of measured dichroic curves and calculated transmission of glass and AR coatings, solid colours). Bottom: filter transmission (dashed) and total calculated instrument efficiency (solid), including filters and detectors but excluding telescope and atmosphere.

down to the Nyquist sampling limit of 0.55 arcsec, defined by the plate scale of $0.276 \operatorname{arcsec}$ pixel $^{-1}$. Optical distortion (pincushion) is small $(<1 \%)$ and, more importantly, very similar for all three channels $( \pm 0.25 \%)$. This ensures that small identical windows can be used when reading out the same region of the FoV from the three cameras. All lenses have broad-band anti-reflection coatings with an average reflectivity below $1 \%$ to increase throughput. To ensure good $u$ efficiency, the collimator and the $u$ camera only make use of glasses with very high UV transmission.

The guiding camera is a commercial $35-\mathrm{mm}$ reflex camera objective (Carl Zeiss Planar $T^{*} 1,4 / 50$ ), with a focal length of $f=50 \mathrm{~mm}$ and an aperture of $f / 1.4$. This camera provides a FoV that is slightly larger than the science field on an ST-1603ME CCD camera (SBIG, USA) with $1020 \times 1530$ $9-\mu \mathrm{m}$ pixels. Unlike the science cameras, the guiding detector does not have a field flattener. Hence, image quality degrades substantially towards the edges of the field.

\subsection{Mechanical design}

MAIA is installed on the instrument rotator at the Nasmyth B focal station of the Mercator telescope (Fig. 5). The 3D drawings in Fig. 6 show the mechanical layout of MAIA. The structural design of the instrument is based on a rigid aluminium box

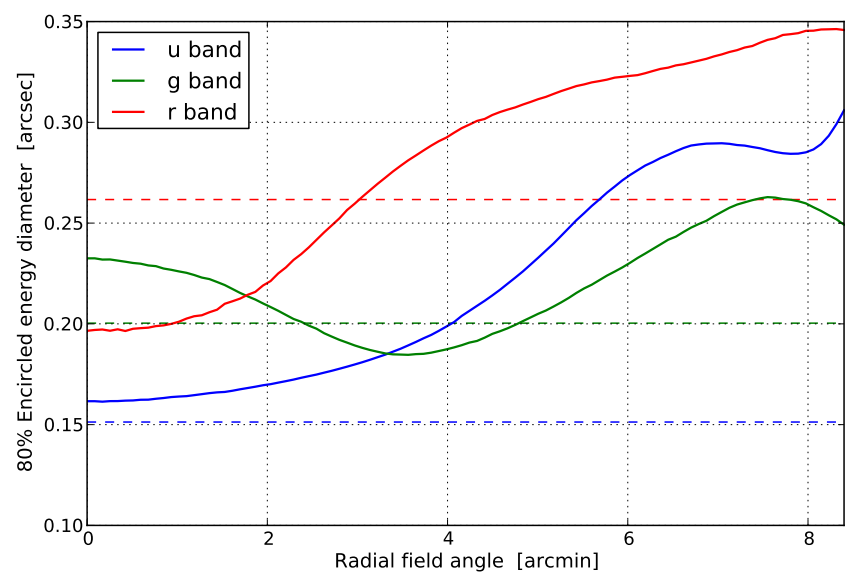

Fig. 4. Calculated $80 \%$ encircled energy diameters over the FoV for each channel; the dashed lines indicate the diffraction limit at the central wavelength.

that holds all the optical subsystems (cameras, collimator, beam splitters). This ensures that the relative positions of the optical elements are very stable. In any orientation, the relative drift between the three channels is limited to just a few detector pixels. Homogeneous temperature changes will lead to isomorphic 


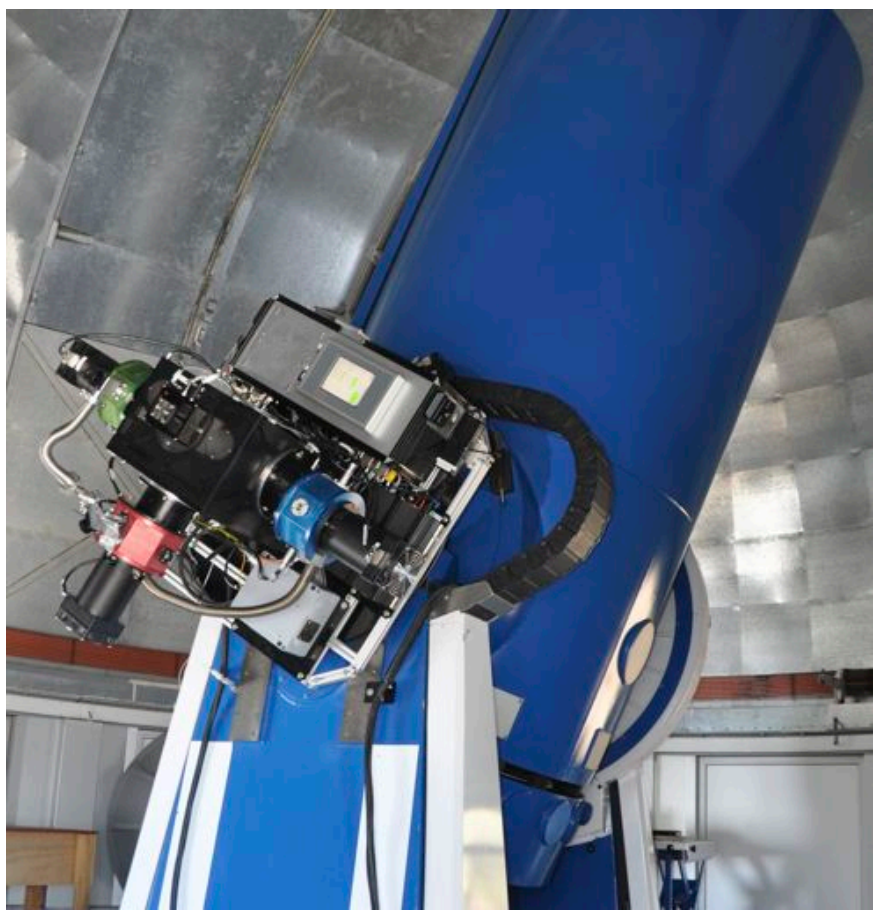

Fig. 5. Picture of MAIA mounted at the Nasmyth focus of the Mercator telescope.

expansion of the aluminium box. This will not affect the angular positions of the cameras and the beam splitters with respect to the collimated beam, hence thermal miss-alignment between the three channels is avoided. Precise machining of the interfaces to the optical subsystems limits the number of required alignments. Access holes for the adjustments of the beam splitter orientation are foreseen. The box is extensively machined from a single block of cast aluminium, reducing its mass to only $15 \mathrm{~kg}$.

The electronic accessories (CCD controller, data-acquisition $\mathrm{PC}$, and control electronics box, housing the PLC with temperature control hardware, cryocooler drivers and power supplies) are not attached to the optics box but mounted on a separate accessories frame that is directly attached to the instrument rotator. This way, their weight does not load the opto-mechanics of the instrument. An open cable reel is installed between the accessories frame and the rotator. The total weight of MAIA, accessories and cable reel included, amounts to $160 \mathrm{~kg}$. The overall length of the instrument is $750 \mathrm{~mm}$ and it rotates within a 660 -mm radius.

\subsubsection{Lens mountings}

The lenses of the collimator and the three cameras are mounted in four lens barrels. The most critical parameter in the lens mountings is the relative centring of the lens groups in their barrel. In order to avoid image quality degradation, some of the lenses should be centred with a precision of $\sim 0.03 \mathrm{~mm}$ and this precision needs to be maintained over the operational temperature range $\left(0^{\circ} \mathrm{C}\right.$ to $\left.25^{\circ} \mathrm{C}\right)$. Moreover, the instrument should be able to resist storage and air transport conditions $\left(-40{ }^{\circ} \mathrm{C}\right.$ to $+55^{\circ} \mathrm{C}$ ). Different coefficients of thermal expansion (CTE) for the mounting barrel and the glass of the lenses prevents us from limiting the radial play of the lens mounts to $0.03 \mathrm{~mm}$ and hence, a different centring concept is required. Moreover, the optical glasses of the lenses have very different CTEs, ranging from $0.55 \times 10^{-6}$ to $18.9 \times 10^{-6} \mathrm{~K}^{-1}$.
To maintain the lenses centred over an extended temperature range, their radial position is constrained by two pins (thermal compensator pins) at a $90^{\circ}$ angle (Fig. 7). To align the centring of the lenses, the effective length of these pins is adjustable by lockable screws. A third spring-loaded pin at $135^{\circ}$ applies a constant force on the lens, pushing it towards the centre while allowing for differential thermal expansion. Both the length and the material of the thermal compensator pins are chosen in such a way that the thermal expansion of the glass, the lens barrel and the pins, keep all lenses on the same optical axis over a broad range of temperatures. We use aluminium, stainless steel or Invar as materials for these pins, depending on the CTE of the lens (Vandersteen 2012).

\subsubsection{Cryocooler vibrations}

Free-piston Stirling coolers provide a compact and lowpower alternative over conventional liquid nitrogen or Joule-Thompsom (CryoTiger) cooling. However, the strong vibrations of the cooling engine can prohibit their application in astronomical instrumentation. With the absence of a viable alternative (Sect. 4.1.2), we decided nevertheless to use Stirling cooling for MAIA. The piston of a Stirling cooler is driven by a linear motor running at $60 \mathrm{~Hz}$. A resonating vibration absorber, precisely tuned to the cooler frequency, is mounted on the backside of the cooler to absorb the bulk of the harmonic disturbances. We measured that this absorber effectively reduces vibrations at $60 \mathrm{~Hz}$ to less than $10 \%$ of their initial amplitude.

Laboratory measurements showed that the remaining cooler vibrations had no detrimental effect on image movement or image quality. However, after installation of MAIA on the telescope, significant vibrations of the telescope structure appeared. These vibrations caused unacceptable oscillations with amplitudes of up to 10 arcsec. Spectral analysis of accelerometer measurements on the instrument and the telescope showed that almost all vibrational energy is contained within very narrow-band signals at $60 \mathrm{~Hz}$ and its higher harmonic frequencies (Fig. 9). The narrow-band nature of the telescope vibrations indicate that they are the result of direct coupling to the Stirling cooler, rather than the excitation of a resonant mode in the telescope structure. Although adding structural damping to this system may intuitively be thought to attenuate vibrations, structural damping is more effectively used to attenuate wide-band signals (Den Hartog 1985). Therefore, we mechanically decoupled the cooler from the rest of the instrument by mounting four leaf springs between cooler and cryostat (Fig. 8). A thin flexible bellows forms the vacuum interface between both parts. These springs create a low-pass mechanical filter that will no longer transmit vibrations of frequencies that are substantially higher than the eigenfrequency of the system.

The eigenfrequency or resonance frequency $f_{\text {res }}$ of a springmass system with $m$ the mass of the cryocooler and $k$ the total spring constant of the leaf springs and the vacuum bellows, equals:

$f_{\mathrm{res}}=\frac{1}{2 \pi} \sqrt{\frac{k}{m}}$.

To obtain a low eigenfrequency, a spring with small stiffness is needed. However, the spring should be stiff enough to resist the force exerted by the vacuum inside the cryostat $(\sim 160 \mathrm{~N}$ at the altitude of the observatory) and to limit the axial and angular excursions of the cooler due to gravity when rotating the instrument. These constraints set a lower limit on $k$ of $33500 \mathrm{~N} \mathrm{~m}^{-1}$. 

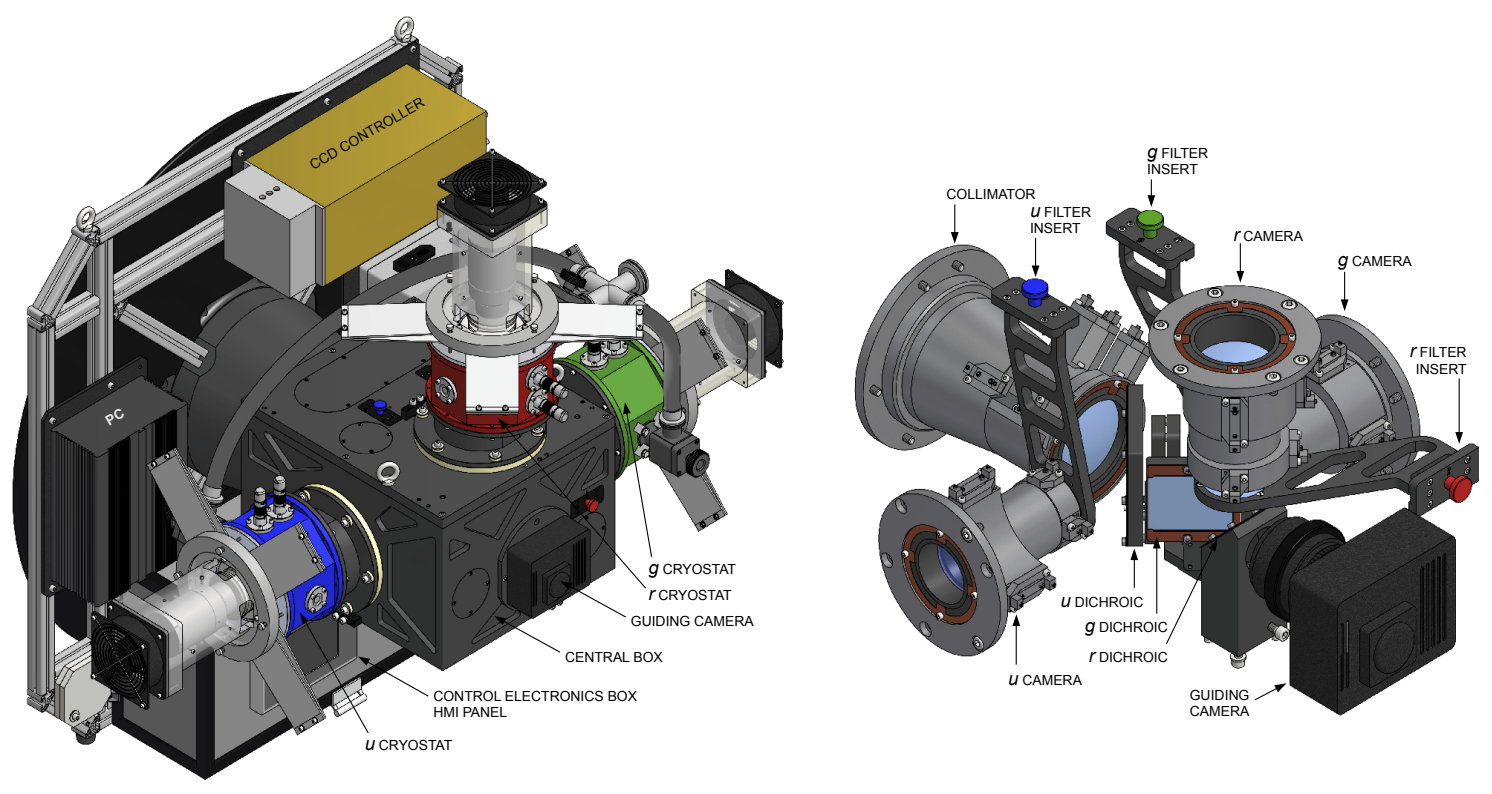

Fig. 6. Left: 3D drawing of complete instrument, the colours of the three cryostats correspond to their respective wavelength bands. Right: internal view of optical subsystems in the central aluminium box.

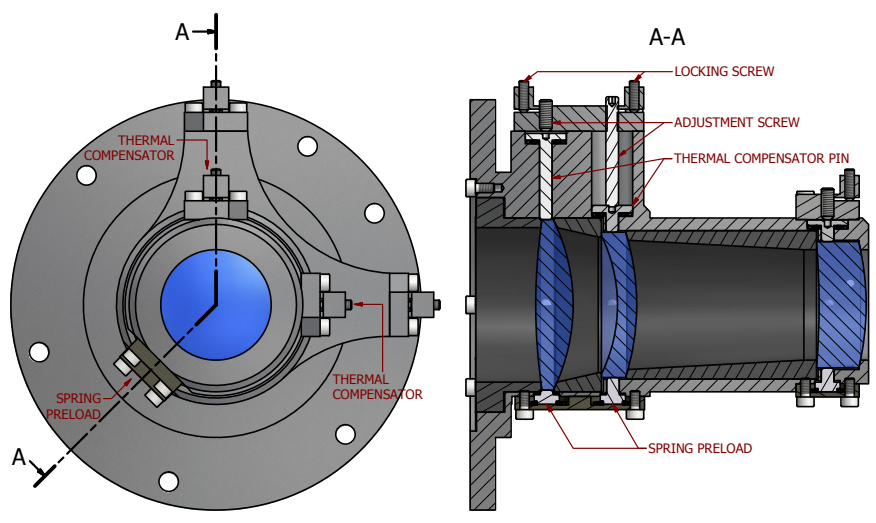

Fig. 7. Front view and longitudinal section view of camera barrel; the thermal compensator pins and the spring preload constrain all lenses (blue) around the optical axis, located at the thermal centre of the system.

To further reduce $f_{\text {res }}$, we increase $m$ by adding some extra mass to the cooler (initial weight: $\sim 4 \mathrm{~kg}$ ). Each cooler receives a stainless steel mounting ring of different weight $(2.2,3.2$ and $4.5 \mathrm{~kg}$ for $r, u$ and $g$ ), resulting in different resonance frequencies for each cooler $(\sim 12,11$, and $10 \mathrm{~Hz}$ for $r, u$ and $g)$, in order to avoid resonant coupling between the three spring-mass systems.

Besides $m$ and $k$, the damping ratio $\zeta$ (a unitless measure that describes the oscillations' decay rate after a disturbance) is a third parameter that characterises the transmission at a given frequency. Large damping results in a small resonance peak at the eigenfrequency but also in a reduced roll-off slope of the low-pass filter and thus, maybe a bit counterintuitively, less efficient attenuation at higher frequencies. We did not encounter any resonance problems, so we tried to use as little damping as possible ( $\zeta \simeq 0.005$, a typical value for undamped metals).

To test the performance of the vibration isolation, we measured the vibrational accelerations of the instrument and the telescope with and without the spring isolators installed. Figure 9 shows a typical vibration spectrum of the $r$ cooler (this is a worst

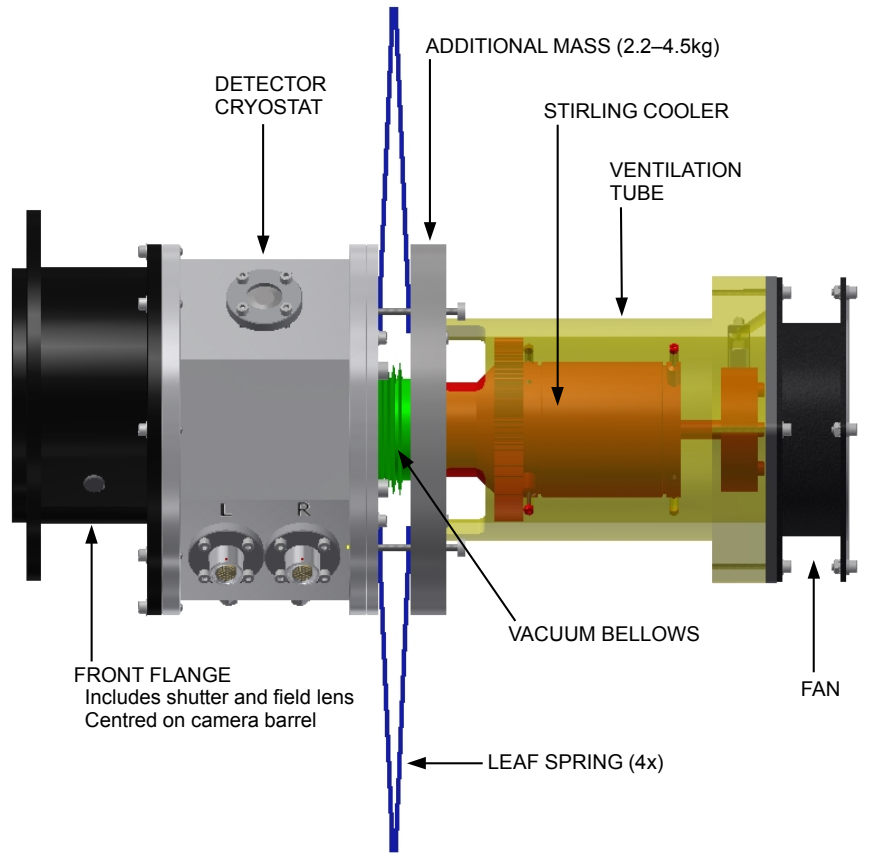

Fig. 8. Drawing of a MAIA cryostat with spring-mounted Stirling cooler. The cooler is fixed in the ventilation tube (drawn transparently for clarity).

case because $r$ has the smallest extra mass and thus the highest eigenfrequency). The accelerations were measured on the instrument along the axis of the piston movement. Measurements at different locations and in different directions give similar results but with smaller amplitudes. The spring isolators reduce the harmonic peaks by several orders of magnitude. At the two main frequencies $(60 \mathrm{~Hz}$ and $420 \mathrm{~Hz})$, this reduction amounts to a factor of 500 and 800 respectively. While not really troublesome, broadband noise is also reduced by an order of magnitude at higher frequencies. After the installation of the vibration 


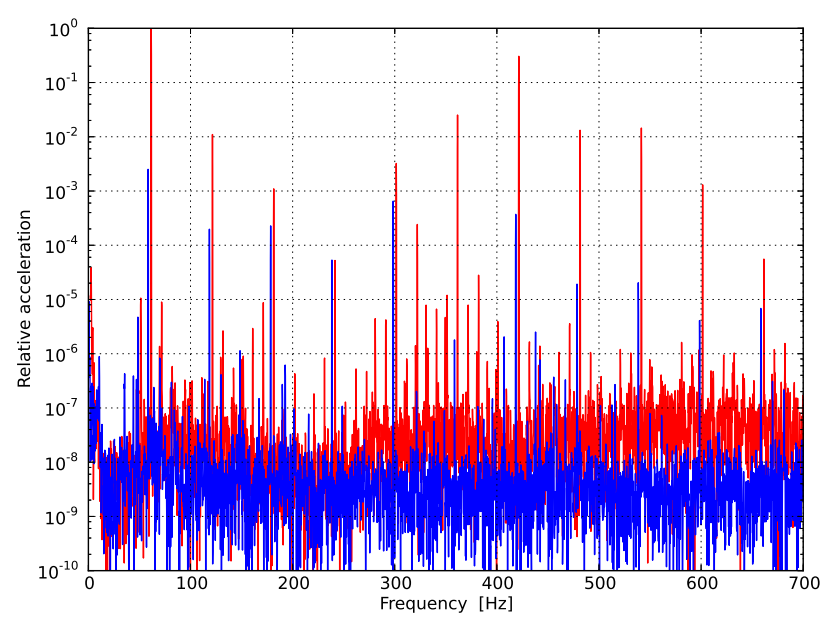

Fig. 9. Instrument radial vibration spectrum without (red) and with (blue) mass-spring decoupling between Stirling cooler and cryostat. Curves are translated by +1 and $-1 \mathrm{~Hz}$ for clarity. Most pronounced vibration peaks occur at multiples of $60 \mathrm{~Hz}$.

isolation, we could no longer detect any perceivable effect on image quality when switching the coolers on or off.

\section{Instrument performance}

\subsection{Efficiency}

To determine the throughput of MAIA on the Mercator telescope, we measured the photometric zero points for all three channels from a series of standard-star exposures at different air masses. These zero points correspond with the magnitude of a source that would give one detected photon per second outside the atmosphere. The results are given in Table 3, together with the typical atmospheric extinction in each wavelength band. From these exposures, we also calculated the total efficiency of MAIA, excluding the telescope mirror reflectivity. It should be noted that these zero-point measurements were performed with a telescope that had a five-year old aluminium mirror coating. We estimated the mirror reflectivity at $83 \%$ or less than $60 \%$ for three reflections. A fresh layer of aluminium on all three telescope mirrors could increase the actual zero points by 0.25 mag.

In $g$ and $r$, the throughput corresponds with our expectations but not in $u$. This is mainly due to the low performance at short wavelengths of the anti-reflection coating on the detector. Since replacing the $u$ detector is not an option, we do not foresee an improvement for this in the near future. However, we also suspect that the transmission of one or more of the MAIA lenses might be substantially below specifications. We are still investigating this and hope to achieve a substantial increase of the $u$ throughput by replacing poorly performing optical elements.

\subsection{Imaging performance}

Laboratory measurements of the image quality of MAIA without the telescope showed a point spread function with a FWHM of less than $17 \mu \mathrm{m}$ or 0.35 arcsec over most of the FoV. This compares well with the theoretical maximum $80 \%$ encircled energy diameters shown in Fig. 4. These FWHM values should not degrade excellent seeing of 0.6 arcsec by more than 0.1 arcsec. As the collimator was optimised in combination with the Mercator telescope optics, performance at the telescope is
Table 3. MAIA measured and calculated peak efficiency (instrument and detectors without telescope), zero points (including telescope) and typical atmospheric extinction magnitudes at the Mercator telescope.

\begin{tabular}{lcccc}
\hline \hline Band & \multicolumn{2}{c}{ Efficiency (\%) } & Zeropoint & Atm. extinction \\
& meas. & calc. & (mag) & (mag) \\
\hline$u$ & 8.5 & 21 & 21.0 & 0.60 \\
$g$ & 51 & 51 & 24.0 & 0.12 \\
$r$ & 49 & 53 & 23.7 & 0.09 \\
\hline
\end{tabular}

expected to be at least as good. However, the first MAIA observing runs suffered from poor observing conditions and bad seeing. Moreover, the overall image quality was further degraded due to non-perfect alignment of the telescope optics. As a result, image quality and spatial resolution have been severely limited up to now and hence, we could only prove that MAIA on the Mercator telescope is at least capable of producing 0.9$\operatorname{arcsec}(\mathrm{FWHM})$ images in all three channels. The telescope misalignment is currently being corrected. Under more favourable atmospheric conditions, we expect to show substantially better imaging performance.

All three cameras have to use the same window dimensions and locations. To reduce read-out time, windows preferably are small, hence, it is important that the individual FoVs of the three cameras precisely coincide. The initial co-alignment of the three cameras is better than \pm 5 pixels in the centre of the FoV. Due to different distortion by the optics of each camera $( \pm 0.25 \%)$, an additional error of 4 pixels can occur at the furthest edge of the detector. Differential gravitational flexure when rotating the instrument causes an additional drift of at most 3 pixels. Hence, a total shift of up to 12 pixels between the three cameras has to be taken into account when defining the window size. Typical windows, assuming a worst-case co-alignment error, allowing for plenty of sky pixels and depending on cycle-time requirements, consist of 60-120 complete detector rows.

\subsection{First results}

To illustrate the data that can be obtained with MAIA we show a $3.5 \mathrm{~h}$ light curve of the high-amplitude hybrid subdwarf B star pulsator Balloon 090100001 (hereafter referred to as Balloon, Oreiro et al. 2004, $V=12.3$ ) in Fig. 10. The observations were performed during a commissioning run in October 2012 using $15 \mathrm{~s}$ integration times.

Balloon's highest amplitude pulsation mode at $2807.5 \mu \mathrm{Hz}$ (356 s) is known to be the fundamental radial mode (Baran et al. 2005). It is clear from the figure that the pulsation amplitude is largest in the $u$, and smallest in the $r$ band, exactly as is expected for a radial $p$-mode (see e.g. Randall et al. 2005). Unfortunately, the target has strong pulsation modes at 2823.2, 2824.8 and $2823.3 \mu \mathrm{Hz}$ (Baran et al. 2008), which are close to the fundamental. Beating between these modes and the fundamental mode explains the reduced variability amplitude at the start of the MAIA observations. The dataset we obtained is not long enough to resolve the beating modes and hence does not allow us to reliably compare the pulsation amplitudes in the different bands. Detailed analyses of longer observation runs on other sdB pulsators will be presented in upcoming papers. 


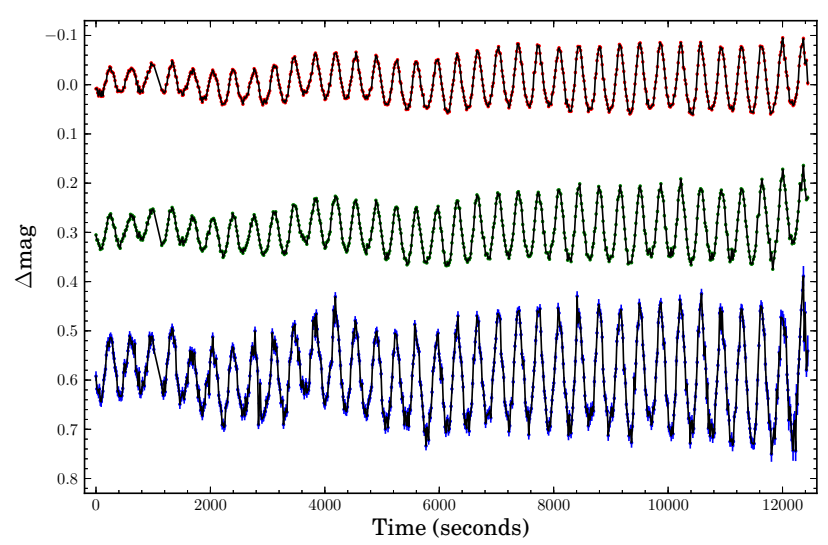

Fig. 10. MAIA light curve of Balloon taken with $15 \mathrm{~s}$ integration times. The different colour bands (from top to bottom, $r$ in red, $g$ in green and $u$ in blue) are offset by 0.2 mag for clarity. The amplitude of the variations in $u$ is clearly higher than the variation in $g$ and $r$, as can be expected for a radial pulsation mode.

\section{Conclusions}

We have successfully built and commissioned MAIA, a threechannel imager capable of producing high-speed three-colour light curves for variable star research, with specific emphasis on pulsation mode identification capability for asteroseismology. With this instrument, we have put the detectors that were developed for the Eddington space mission, to practical use in the science field for which they were originally designed. MAIA can increase the efficiency of the Mercator telescope for multi-colour photometry by a factor of up to six: a factor of three because of the simultaneous observations in three colour bands and another factor of two thanks to the absence of dead time during detector read out in case of fast-cadence observations. Furthermore, with a FoV of $9.4 \times 14.1 \operatorname{arcmin}^{2}$, MAIA has more than doubled the field coverage of the Mercator telescope compared to the former MEROPE II camera.

A limiting property of MAIA is the throughput in $u$ band that falls almost a magnitude short with respect to the specifications, mainly due to the non-optimised anti-reflection coating of the detector. This non-compliance is still the subject of further study, with the aim of at least a partial recovery of the $u$-band throughput in the future.

The combination of a dedicated telescope like Mercator and the MAIA instrument offers unique capabilities for the study of variable stars, and in particular for short-period pulsators. The instrument will also be used for follow-up observations of targets studied from MOST, CoRoT and Kepler space-based whitelight photometry for which mode identification is lacking and/or insufficient frequency precision occurs, preventing in-depth seismic modelling.

We plan to present the full details of the commisioning data of a selected pulsating $\mathrm{sdB}$ star, along with a description of the data reduction software, in a forthcoming paper. On completion of the commissioning and software pipeline, MAIA will be offered to the community on a collaborative basis keeping in mind the overall scheduling and partnership requirements of the Mercator telescope.
Acknowledgements. This research was based on funding from the European Research Council under the European Community's Seventh Framework Programme (FP7/2007-2013)/ERC grant agreement no 227224 (PROSPERITY) and from the Fund for Scientific Research of Flanders (FWO), grant agreements G.0410.09 and G.0470.07, and the Big Science program. The CCDs of the MAIA camera were developed by e2v in the framework of the Eddington space mission project and are owned by the European Space Agency; they were offered on permanent loan to the Institute of Astronomy of KU Leuven, Belgium, with the aim to build and exploit an instrument for asteroseismology research to be installed at the $1.2 \mathrm{~m}$ Mercator telescope at La Palma Observatory, Canary Islands. Conny Aerts is grateful to Giuseppe Sarri and Fabio Favata for their support and help in the practical implementation of the ESA loan agreement with KU Leuven. We thank Simon Tulloch for his help in the optimisation of the detector control, Tibor Agócs for his valuable comments on the optical design of MAIA and Robin Lombaert for transporting the instrument to La Palma.

\section{References}

Aerts, C., Christensen-Dalsgaard, J., \& Kurtz, D. W. 2010, Asteroseismology, Asteroseismology, Astronomy and Astrophysics Library (Springer) Auvergne, M., Bodin, P., Boisnard, L., et al. 2009, A\&A, 506, 411 Baran, A., Pigulski, A., Kozieł, D., et al. 2005, MNRAS, 360, 737 Baran, A., Pigulski, A., \& O’Toole, S. J. 2008, MNRAS, 385, 255 Beck, P. G., Montalban, J., Kallinger, T., et al. 2012, Nature, 481, 55 Bedding, T. R., Mosser, B., Huber, D., et al. 2011, Nature, 471, 608 Charpinet, S., Van Grootel, V., Fontaine, G., et al. 2011, A\&A, 530, A3 Christensen-Dalsgaard, J. 2002, Rev. Mod. Phys., 74, 1073

Davignon, G., Blecha, A., Burki, G., et al. 2004, in SPIE Conf. Ser. 5492, eds. A. F. M. Moorwood, \& M. Iye, 871

Degroote, P., Aerts, C., Baglin, A., et al. 2010, Nature, 464, 259

Deheuvels, S., García, R. A., Chaplin, W. J., et al. 2012, ApJ, 756, 19

Den Hartog, J. 1985, Mechanical Vibrations, Dover Books on Engineering (Dover Publications)

Dhillon, V. S., Marsh, T. R., Stevenson, M. J., et al. 2007, MNRAS, 378, 825

Favata, F., Roxburgh, I., \& Christensen-Dalsgaard, J. 2000, in The Third MONS

Workshop: Science Preparation and Target Selection, eds. T. Teixeira, \& T. Bedding, 49

Gilliland, R. L., Brown, T. M., Christensen-Dalsgaard, J., et al. 2010, PASP, 122, 131

Hu, H., Dupret, M.-A., Aerts, C., et al. 2008, A\&A, 490, 243

Huat, A.-L., Hubert, A.-M., Baudin, F., et al. 2009, A\&A, 506, 95

Leach, R. W., \& Low, F. J. 2000, in SPIE Conf. Ser. 4008, eds. M. Iye, \& A. F. Moorwood, 337

Lumb, D. H., \& Favata, F. 2003, in SPIE Conf. Ser. 5170, ed. D. R. Coulter, 177 Neiner, C., Floquet, M., Samadi, R., et al. 2012, A\&A, 546, A47

Oreiro, R., Ulla, A., Pérez Hernández, F., et al. 2004, A\&A, 418, 243

Østensen, R. H. 2010, Astron. Nachr., 331, 1026

Østensen, R. H. 2013, in 18th European White Dwarf Workshop., eds. J. ski

Krzesiń, G. Stachowski, P. Moskalik, \& K. Bajan, ASP Conf. Ser., 469, 3

Østensen, R. H., Green, E. M., Bloemen, S., et al. 2010, MNRAS, 408, L51

Pablo, H., Kawaler, S. D., Reed, M. D., et al. 2012, MNRAS, 422, 1343

Pápics, P. I., Briquet, M., Baglin, A., et al. 2012, A\&A, 542, A55

Pessemier, W., Deconinck, G., Raskin, G., Saey, P., \& Van Winckel, H. 2012, in SPIE Conf. Ser., 8451, 84512

Randall, S. K., Fontaine, G., Brassard, P., \& Bergeron, P. 2005, ApJS, 161, 456 Raskin, G., Burki, G., Burnet, M., et al. 2004, in SPIE Conf. Ser. 5492, eds. A. F. M. Moorwood, \& M. Iye, 830

Raskin, G., van Winckel, H., Hensberge, H., et al. 2011, A\&A, 526, A69

Reed, M. D., Baran, A., Quint, A. C., et al. 2011, MNRAS, 414, 2885

Unger, R., \& Keiter, D. 2004, AIP Conf. Proc., 710, 1404

Van Grootel, V., Charpinet, S., Fontaine, G., et al. 2010, ApJ, 718, L97

Vandersteen, J. 2012, Ph.D. Thesis, KU Leuven, Belgium,

http://fys.kuleuven.be/ster/pub/jeroen-vandersteen/ phd-thesis-jeroen-vandersteen-1

Vandersteen, J., Raskin, G., Agócs, T., et al. 2010, in SPIE Conf. Ser., 7735, 77353B

Vos, J., Østensen, R. H., Degroote, P., et al. 2012, A\&A, 548, A6

Walker, G., Matthews, J., Kuschnig, R., et al. 2003, PASP, 115, 1023 